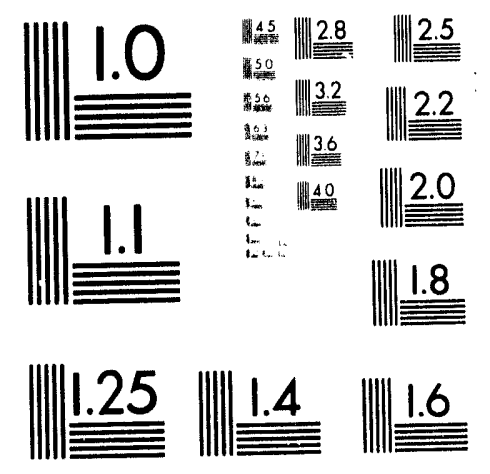



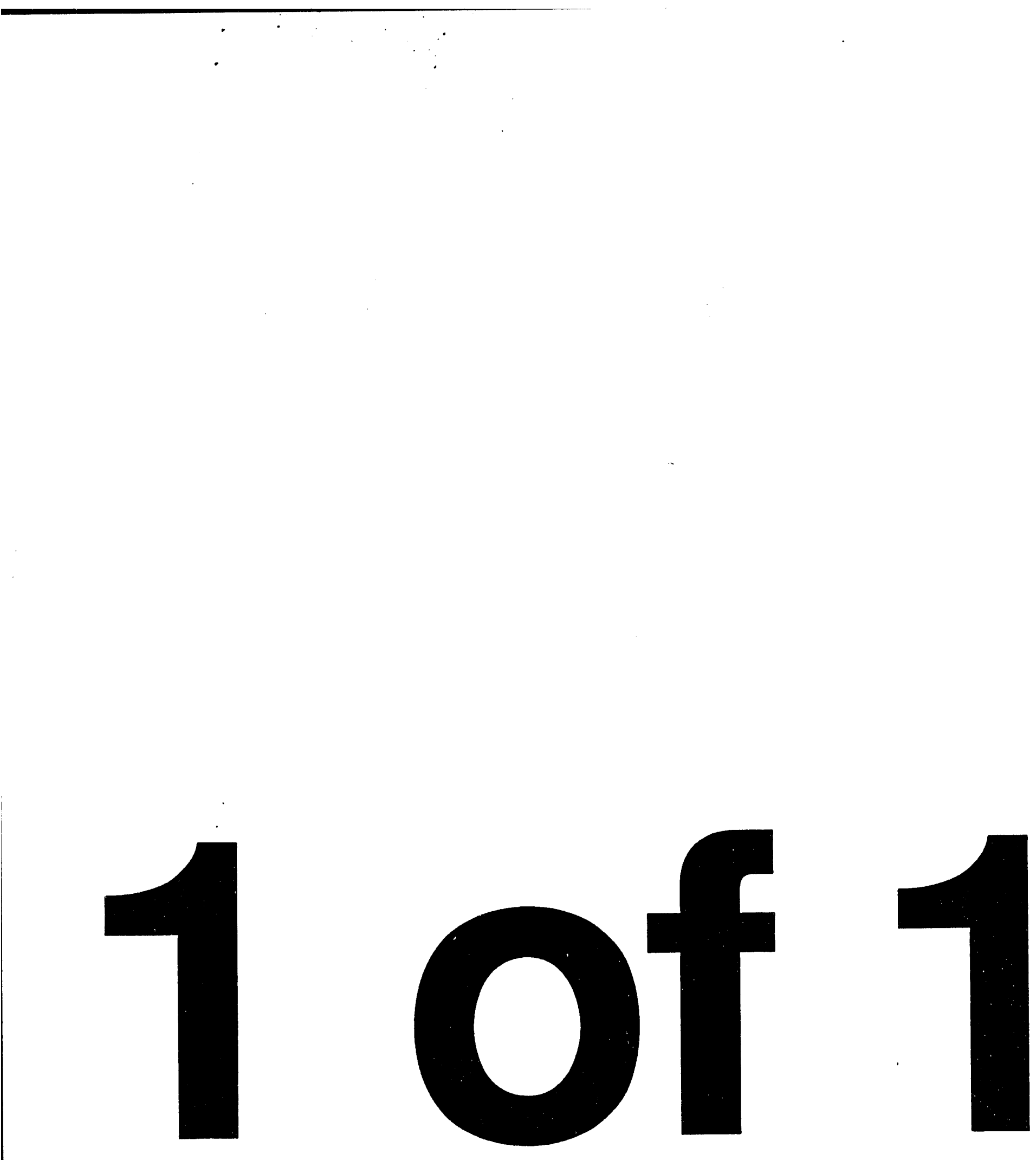
LBL-33706

\title{
Data Management Tools for Genomic Applications: A Progress Report
}

\author{
Victor M. Markowitz and I-Min A. Chen \\ Information and Computing Sciences Division \\ Lawrence Berkeley Laboratory \\ University of California \\ Berkeley, CA 94720
}

September 1993

This work was supported by the Office of Health and Environmental Research Program of the Office of Energy Research, U.S. Department of Energy under Contract DE-AC03-76SF00098. 


\title{
Data Management Tools for Genomic Applications: A Progress Report *
}

\author{
Victor M. Markowitz and I-Min A. Chen \\ Information and Computing Sciences Division \\ Lawrence Berkeley Laboratory, Berkeley, CA 94720
}

\begin{abstract}
We report in this paper on the development of data management tools that allow scientists to construct and manipulate genomic databases in terms of application-specific objects and protocols. We are developing tools for specifying genomic database structures, as well as for entering, changing, maintaining, browsing and querying data in genomic databases. These tools are based on the Object-Protocol Model (OPM) developed by us and target commercial relational database management systems which are widely used in molecular biology laboratories. OPM allows ccientists to interact with genomic databases in terms of their own frame of reference, naniely genomic objects and protocols. Databases developed using the data managiment tools are easier to use, manage, and adapt.
\end{abstract}

\section{Introduction}

The information controlling the development of biological organisms is encoded in their genome in the form of polymeric molecules known as DNA. DNA information is encoded as a sequence of nucleotides. Regions of the DNA called genes specify the information for protein molecules. In higher organisms (yeast, plants, animals, humans) the DNA is organized into several linear chromosomes.

Several projects are attempting to determine the complete DNA sequence of various organisms. These projects require databases for managing DNA data and related information. Typically, the structure of a genomic database can be modeled in terms of objects characterized by (having) attributes that take values from a domain (set of values); objects that share common attributes can be organized (classified) into homogeneous sets of objects. For example, consider the contig maps used in determining the complete DNA sequence of various

-Issued as Techrical Report LBL-33706. This work is supported by the Office of Health and Environmental Research Program of the Office of Energy Research, U.S. [lepartment of Energy under Contract DE-AC03-76SF00098. 
organisms, and consisting of ordered DNA fragments ${ }^{1}$. Contig maps can be modeled as objects that have attributes such as contig id, orner (representing owners of contig maps), and (tragment, position) (representing component fragments and their positions in contig maps); similarly, fragments can be modeled as objects that have attributes such as fragment id and orner.

Genomic databases also contain data on protocols representing experimental laboratory procedures. Given an input, a protocol instance (i.e., an elementary experiment) results in an output. Protocols often involve a series of subprotocol steps. The recursive specification of protocols in terms of component subprotocols is called protocol expansion. Protocol expansion reveals the composition of component subprotocols and/or alternative ways of performing the protocol. For example, consider a construct protocol for constructing contig maps of ordered DNA fragments: such a protocol is applied on DNA fragments (input) and result in contig maps (output). Protocol construet can be expanded into two alternative protocols, overlap and constraint, both followed by protocol assemble: protocol overlap compares two DNA fragments using a computer program, protocol constraint compares manually two DNA fragments according to certain constraints, and protocol assemble assembles DNA fragments into a contig map according to information in the connection tables regarding possible connecting positions of two DNA fragments.

Most genomic databases developed in the past few years use commercial relational database management systems (DBMSs). Relational DBMSs do not provide constructs for representing directly genomic-specific objects and protocols. These objects and protocols are usually represented in relational databases by several disconnected tuples scattered among multiple tables, logically tied to gether by primary key-foreign key references. Such representations are not only hard to comprehend, but also entail the development of large procedures for assembling data on application-specific objects from (i.e., by joining) several relations. Furthermore, because of the complexity of the relational representations for objects and protocols, the development, maintenance, and modification of such databases are tedious, error-prone, and time-consuming processes.

Data models such as the Extended Entity-Relationship Model (EERM) [10] and the Semantic Data Model (SDM) [4] provide constructs for modeling objects, sets of objects, and object associations, and therefore are better suited than relational DBMSs for specifying the structure of genomic databases. For example, in EERM atomic objects called entities are classified into entity-sets, and are qualified by attributes that take values from value-sets. Associations of entities are modeled as relationships classified in relationship-sets. EERM has a generalization mechanism that allows viewing similar (specialization) entity-sets as a single generic entity-set.

We have explored using EERM for describing genomic databases [9], and found that it is too restricted for specifying accurately their object structure. Such restrictions can be overcome by using auxiliary entity-sets and relationship-

\footnotetext{
${ }^{1}$ Since existing technology permits sequencing only fragments of a few hundred nucleotides, chromosomal DNA is cut into smaller fragments, the fragments are propagated as clones, and then assembled into contig maps.
} 
sets. For example, contig maps, fragments and their owners can be represented by three EERM entity-sets called CONTIG_MAP, FRAGMENT, and PERSON, respectively. However, representing that contig maps and fragments can be owned by persons requires an auxiliary entity-set generalizing CORTIG MAP and FRAGMENT, OWHED OBJECT, together with an auxiliary relationship-set, OWNED_BY, associating OWBED_OBJECT with PERSON. Auxiliary constructs do not represent applicationspecific objects and therefore unnecessarily increase the complexity and obscure the semantics of databases.

The need to employ a diversity of continuously evolving mapping and sequencing strategies require facilities for efficiently constructing genomic databases that are easy to use and change. In order to attain the desired level of flexibility and adaptability, we decided to develop data management tools that allow scientists to rapidly construct and manipulate genomic databases in terms of genomic objects and protocols. The underlying data model for these tools is provided by the Object-Protocol Model (OPM) developed by us.

OPM has similarities with other object data models (cf. [5]), especially with SDM [4]. Similar to SDM, in OPM objects are classified into object classes and are qualified by attributes that take values from value classes. Unlike SDM, however, in OPM attributes can be composite, that is, consisting of multiple component simple attributes, and can be associated not only with single value classes, but also with unions of value classes. These constructs allow avoiding the creation of object classes that do not have an application-specific counterpart. Furthermore, unlike other data models (e.g., such as those reviewed in [5] or [6]), OPM provides a protocol class construct for modeling laboratory experiments. A protocol class in OPM can be associated with regular attributes as well as input and output attributes used for specifying input-output protocol connections. OPM also supports a protocol expansion mechanism for specifying a protocol class in terms of component subprotocol classes.

The data management tools we develop will benefit several molecular biology laboratories and genome centers. In particular, our project supports directly tie large-scale sequencing project at University of Washington, Seattle, for characterizing up to six million bases of the human and mouse $\mathrm{T}$-cell receptor loci and the development of the Integrated Genomic Database at the German Cancer Research Center, at Heildelberg.

The rest of the paper is organized as follows. Our approach to developing data management tools is described in section 2. The status of our work is reviewed in section 3 . Section 4 briefly discusses future plans.

\section{Approach}

The data management tools are based on a data model developed by us, the Object-Protocol Molel (OPM). OPM is briefly reviewed below. A complete description of OPM is provided in [1]. 


\subsection{The Object-Protocol Model}

OPM allows describing database structures in terms of objects characterized by attributes taking values from value classes, and classified into object classes. For example, the contig maps mentioned in the previous section can be represented in OPM by object class CONTIG MAP having attributes contig.id, onner, and (fragment, position). Similarly, fragments can be represented by object class FRAGKEIT having attributes fragment id, sequence, length, and orner; and owners can be represented by object class PERSON having attributes person id, name and orns (see figure 1).

Object classes can have subclass-superclass relationships. For example, one can specify a class SCIENTIST as a subclass of PERSON.

Attributes in OPM can be:

1. atomic, such as attribute contigid of object class CONTIG MAP, or composite, that is, consisting of aggregations of atomic attributes, such as attribute (fragment, position) of CONTIG MAP;

2. single-valued, such as attribute person_id of object class PERSON, or multivalued, such as attribute Orns of PERSON;

3. local, such as attribute sequence of object class FRAGMENT, or referential, that is, representing references to other objects, such as attribute orner of FRAGMENT, representing references to PERSON;

4. associated with a single domain, such as attribute name of object class PERSON, or with a union of different domains, such as orns of PERSON whose domain is the union of object classes CONTIG_MAP and FRAGMENT;

5. derived, that is, attributes that have values derived from the values of other attributes using a derivation expression, such as attribute composition, arithmetic expressions, aggregate functions, or attribute inversion; for example, attribute onner of CONTIG MAP in figure 1 is specified as the inverse of attribute orns of PERSON (i.e., the value of orner for a given contig map $m$ is the person whose orns value contains $m$ ).

In addition to objects, OPM supports modeling laboratory protocols. Protocols are classified in protocol classes and can be qualified by both regular and special, input and output, attributes. For example, protocols construct, overlap, constraint, and assemble mentioned in the previous section can be described in OPM by the protocol classes shown in figure 2, where their inputs and outputs are modeled by the object classes shown in figure 1 . Thus, the experiments for constructing contig maps of ordered DNA fragments can be represented by the instances of protocol class CONSTRUCT having an output attribute contigmap representing the result of construct protocols applied on fragments, where fragments are represented by input attribute fragments.

OPM has a protocol expansion mechanism for the recursive specification of protocols in terms of alternative protocols, sequences of protocols, and optional protocols; "or", ",", and "[ ]" are used to denote alternative, sequences 


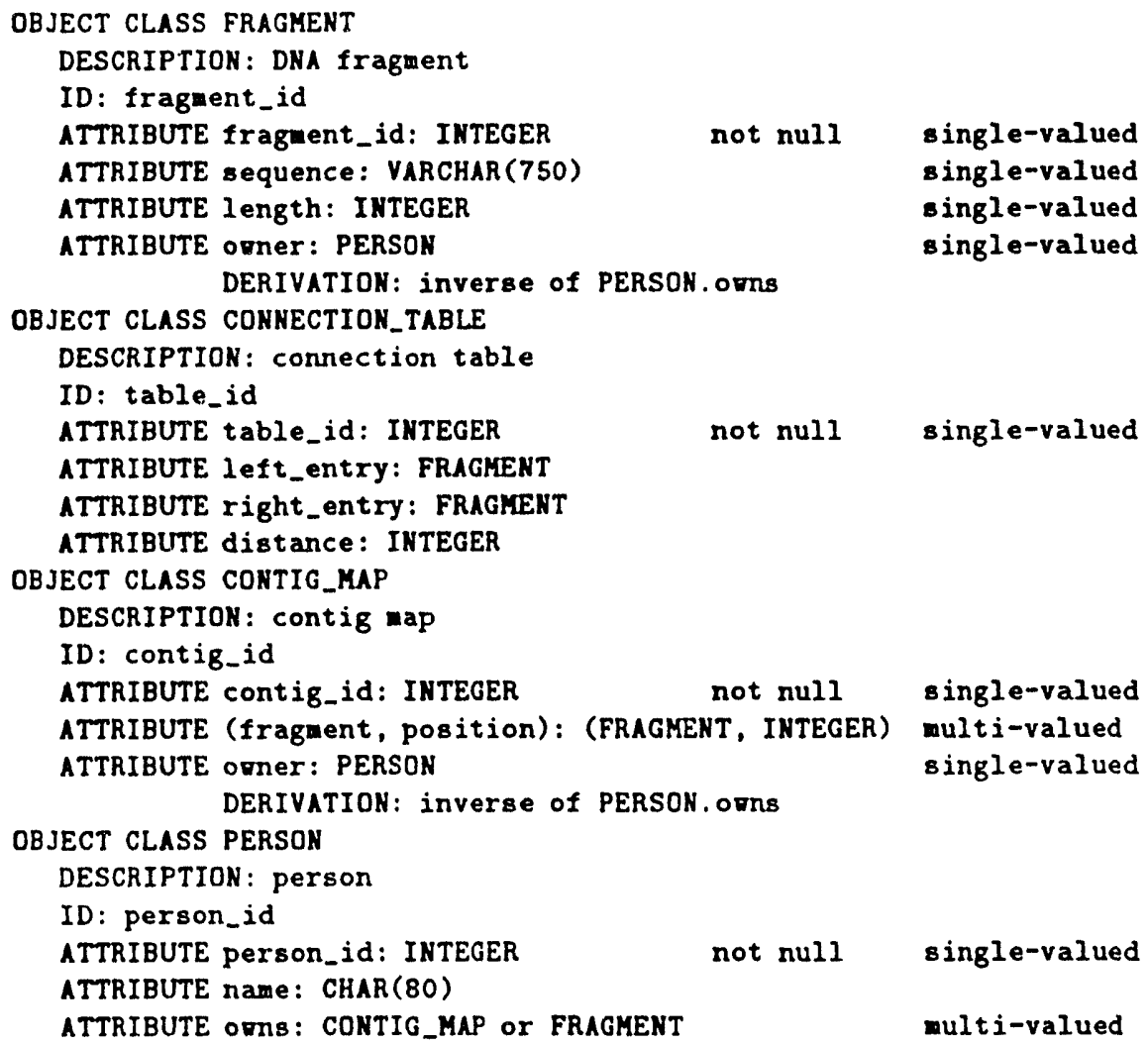

Figure 1: Object Classes Representing the Input and Output for Protocols

of, and optional protocols, respectively, and parentheses are used for specifying complex protocol compositions. For example, consider protocol classes CONSTRUCT, OVERLAP, CONSTRAIKT, and ASSEMBLE shown in figure 2 . The expansion of CONSTRUCT in terms of OVERLAP, CONSTRAINT, and ASSEMBLE is expressed as follows (see figure 2): EXPANSION: (OVERLAP or CONSTRAINT), ASSEMBLE.

Input and output attributes associated with protocols represent the input and output of protocols, respectively, and can be used to express the inheritance of input or output attributes by component subprotocols from their generic protocols and the input-output connection of directly related protocols. Input-output attribute inheritance is expressed using 'input is-a ...' statements (e.g., see attribute fragments of OVERL.AP in figure 2) and 'output is-a ...' statements (e.g., see attribute contig map of ASSEMBLE in figure 2) in the specification of the input and output attributes associated with subprotocols. If a protocol is followed directly by another protocol, then the input of the latter may include some or all of the output of the former. Such input-output 


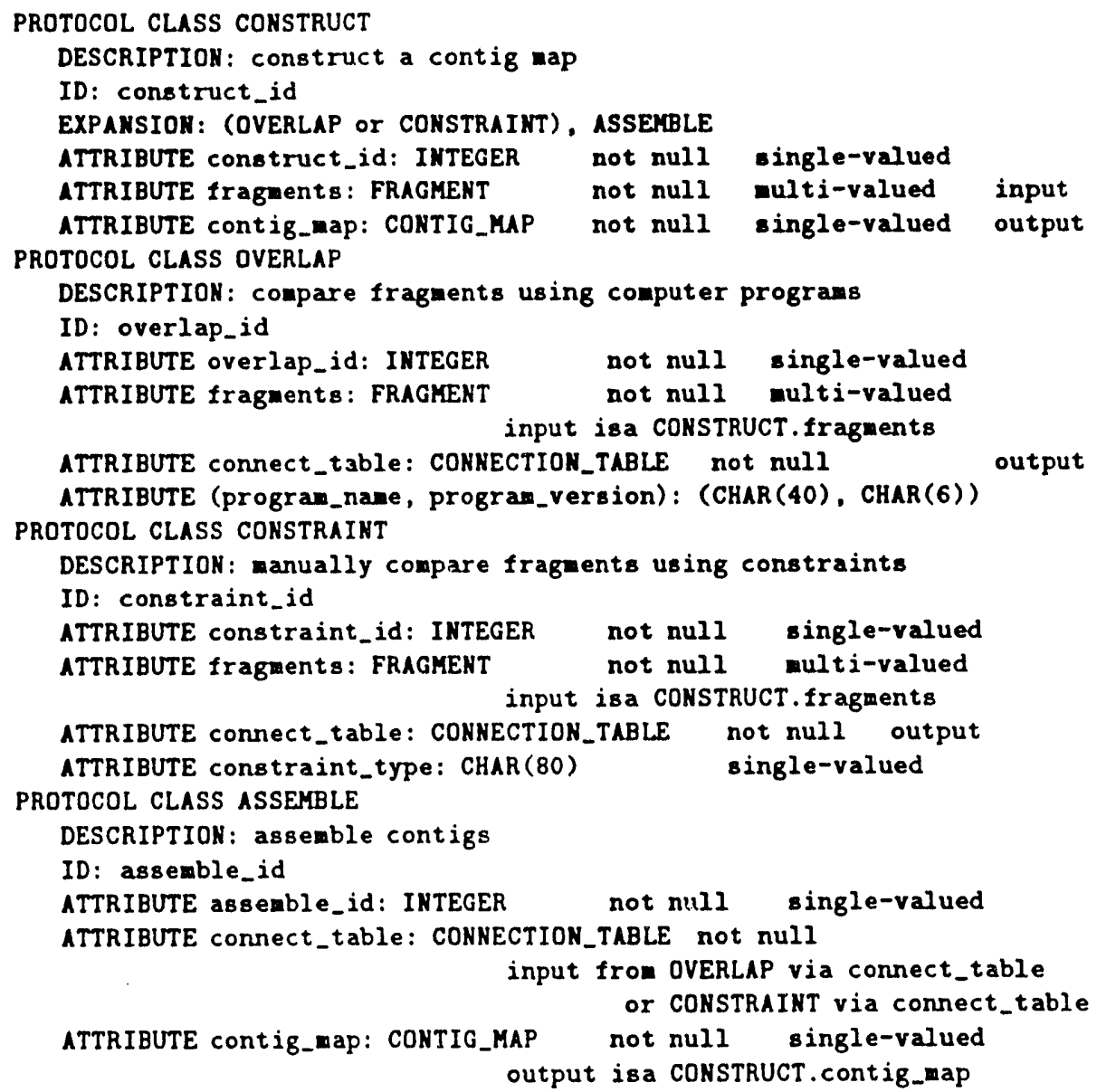

Figure 2: Protocol Classes Representing Protocol Construct and its Components

attribute connections are expressed using 'input from ... via ...' statements (e.g., see attribute connect_table of ASSEMBLE in figure 2) in the specification of input attributes associated with protocols taking their input from other protocols.

\subsection{Data Management Tool Development}

Developing data management tools based on OPM and targeting relational DBMSs, involves mapping OPM constructs and data manipulation operations (retrievals and updates) into relational DBMS constructs and SQL queries. This mapping is very complex because of the discrepancy between the OPM and relational DBMS constructs, but can be simplified by introducing an intermediate EERM level that allows decomposing the OPM to relational DBMS mapping 
into simpler mappings between OPM and EERM, and between EERM and relational DBMS, respectively. The OPM to EERM mapping is easier to develop than the direct OPM to relational DBMS mapping because EERM schemas and queries are specified in terms of objects and object associations, and therefore are inherently more concise and simpler to specify than relational DBMS schemas and queries. Furthermore, EERM schemas and queries are independent of a specific DBMS, and therefore can be used across different DBMS platforms. The EERM version we use is the EERM described in [10], extended with two constructs (unary relationship-sets and a new form of directly associating entitysets) described in [2].

We have developed a mapping of OPM schemas that generates EERM schemas together with queries for constructing OPM objects and protocols from entities and relationships. These queries are expressed in the Concise Object Query Language (COQL) [11], and involve associating a (primary) entity-set with attributes of other (auxiliary) entity-sets and relationship-sets, where the primary entity-set is associated with the auxiliary entity-sets and relationshipsets either directly or via other entity-sets and relationship-sets. Thus, a primary entity-set, its local and inherited attributes as well as the attributes of auxiliary entity-sets and relationship-sets can be specified in COQL using an OUTPUT statement, while the association of a primary entity-set with auxiliary entitysets and relationship-sets can be expressed using CONNECTIONS statements. COQL also allows setting conditions on entity-sets and relationship-sets. Suppose that the contig inaps and their owners mentioned above are represented by entity-sets CONTIG_MAP (with attribute contig_id) and PERSON, connected by relationship-set OWNED BY. Then the following COQL query expresses the association of contig majs with their owners:

$$
\begin{array}{ll}
\text { OUTPUT } & \text { CONTIG MAP: contig id, PERSON; } \\
\text { CONNECTIONS CONTIG MAP OWNED_BY PERSON; END }
\end{array}
$$

In the COQL query above, PERSON is an auxiliary entity-set whose attributes are associated with CONTIG_MAP via relationship-set OWNED_BY.

Regarding the mapping of EERM schemas and queries into relational DBMS schemas and queries, we have developed tools that can automatically carry out the EERM to relational DBMS schema and query mapping. The EERM schema to relational schema mapping is presented in [10] and has been implemented as part of an EERM schema translation tool called SDT [8]. SDT automatically translates EER schemas into schema definitions for several relational DBMSs: Sybase, Ingres, Informix, and Oracle. The DBMS database definitions generated by SDT include procedures (e.g., triggers in Sybase) necessary for maintaining referential integrity and value constraints. The information about schemas and their mapping is subsequently stored in a metadatabase.

The COQL to SQL mapping is described in [11], and has been implemented as part of a COQL translation tool. Based on the metadatabase generated by SDT, the COQL translator maps a COQL query into one or several queries in the SQL dialect of the underlying relational DBMS. The COQL translator has been implemented for Sybase and will be implemented for other relational DBMSs as well. 


\section{Development Status}

In this section we briefly review the status of the OPM data management tools.

\subsection{The OPM Schema Editor}

We have developed a graphical schema editor for interactively specifying, displaying, modifying, merging, and browsing OPM schemas.

The OPM schema editor allows specifying incrementally complex object and protocol structures by providing facilities for defining new schemas, modifying existing schemas, and merging schemas. A schema can be browsed using an Object Classes Listbox that lists in the main window the object classes of the schema (see figure 3). This listbox can be switched into a Protocol Classes Listbox or a Controlled Value Classes Listbox for browsing protocol classes and controlled value classes, respectively.

For an object class selected in the Object Classes Listbox, its connections to other classes (via attributes), and its superclasses and subclasses are displayed in the drawing area of the main window. This graphical display can be also used for browsing a schema by recursively expanding value classes associated with displayed attributes.

An object class can be defined or modified by double clicking on the name of an object class in the listbox or in the drawing area, or by selecting the OPM Object Class option of the Define menu item in the main window. The Object Class Definition window shown in figure 3 illustrates the definition of object class CONTIG MAP. The Define Attribute option in this window allows defining or modifying attributes of the current class.

The Composite Attribute Definition and Component Attribute Definition windows shown in figure 3 illustrate the definition of a composite attribute, namely attribute (fragment, position) of CONTIG MAP. The Attribute Inverse Definition window shown in the same figure allows specifying object cross referencing by defining attributes as inverses of other attributes.

Protocol classes can be browsed, defined or modified in a similar way. For a protocol class selected in the Protocol Classes Listbox, its connections to other classes via attributes, as well as the graphical representation (in a DFD like notation) of its expansion (if any) are displayed in the main window drawing area. A protocol object class can be defined or modified by double clicking on the name of a protocol class in the listbox or in the drawing area, or by selecting the OPM Protocol Class option of the Define menu item in the main window. Figure 4 illustrates the definition of a protocol class (CONSTRUCT) and its subprotocols. The Input/Output Attribute Definition window allows specifying the input and output attributes of protocols. Protocol expansion can be defined or modified using the Protocol Expansion window. The Input From Definition window allows specifying 'input from ... via' connections. For example, in figure 4) attribute connect_table of protocol class ASSEMBLE is specified as: from OVERLAP via connect_table or from CONSTRAINT via connect_table

A schema can be saved in a text file by selecting Generate OPM option of the 


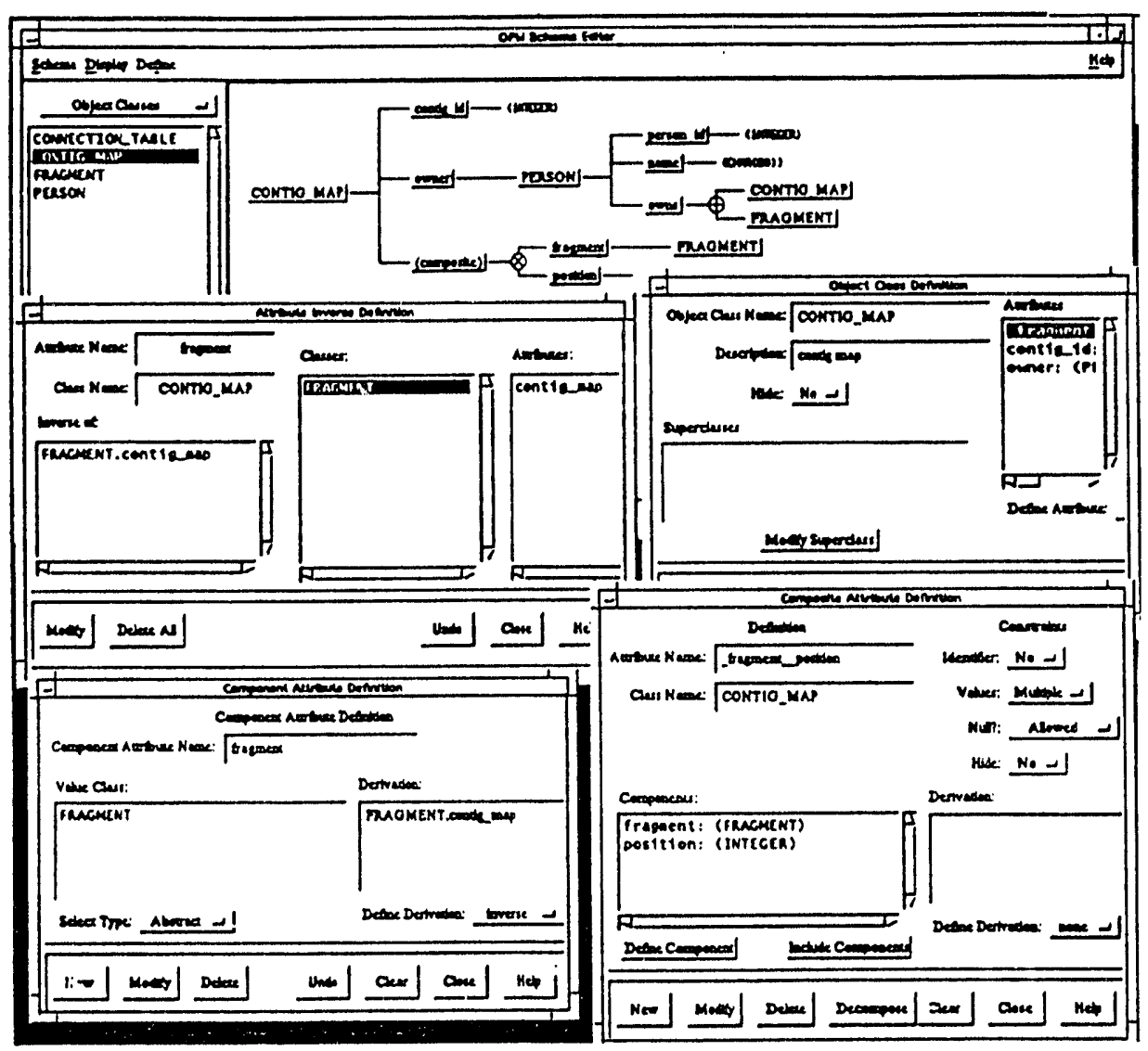

Figure 3: Specifying Object Classes using the OPM Schema Editor

Schema menu item in the main window. This file contains the schema definition in the OPM data definition language, and can be passed to the OPM schema translator described below, for generating the corresponding EER schema and COQL queries.

The OPM schema editor has been implemented on Sun SPARCstations using $\mathrm{C}++$ and the X11 Motif graphical user interface toolkit and is described in [3].

\subsection{The OPM Schema Translator}

Since scientists in most molecular biology laboratories use commercial relational DBMSs (mainly Sybase), the tools we develop target relational DBMSs. Consequently, these tools involve mapping OPM schemas into relational schema definitions and SQL queries that express basic manipulations (retrievals and updates) of OPM objects and protocols. 


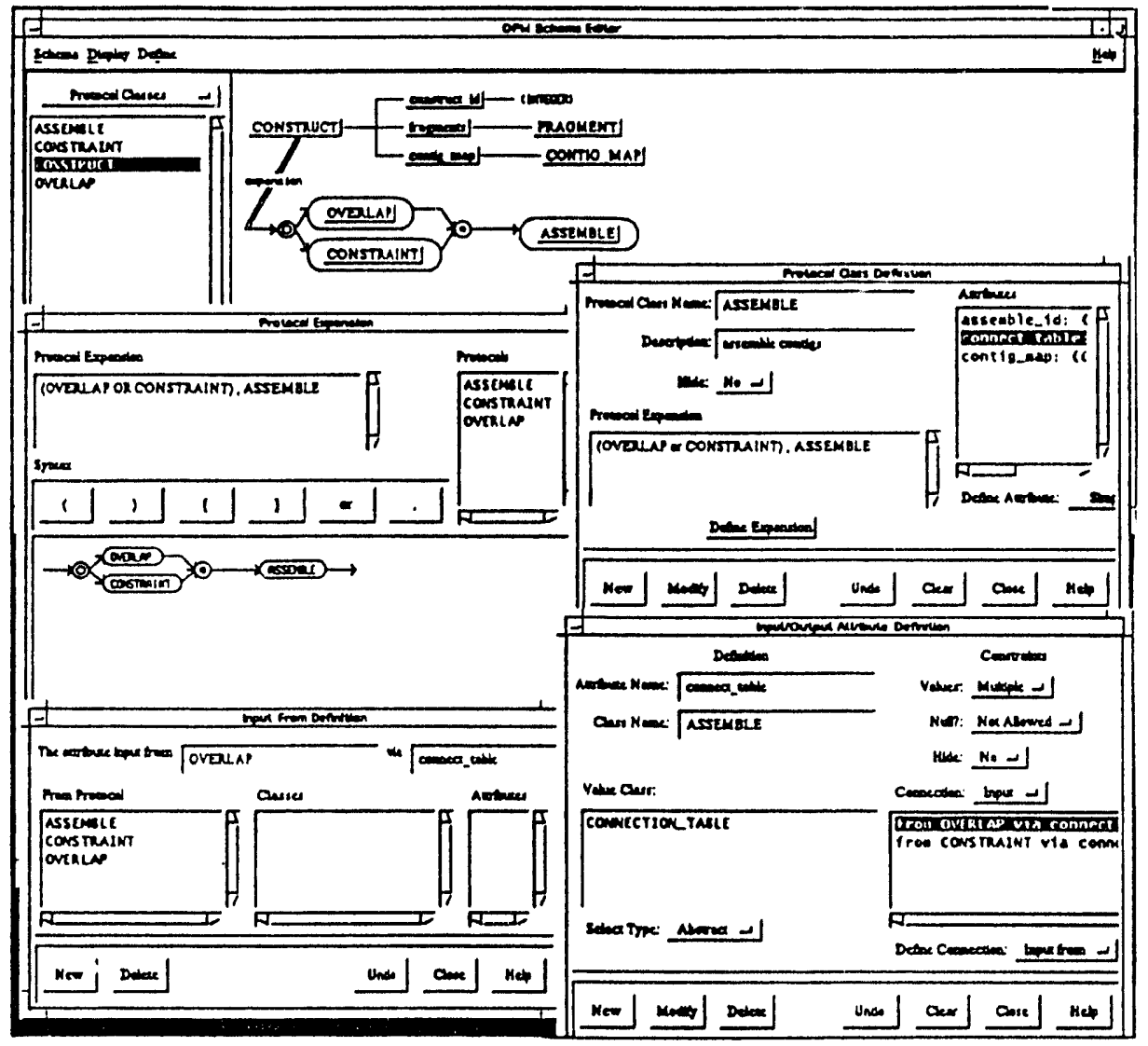

Figure 4: Specifying Protocol Classes using the OPM Schema Editor

As already mentioned above, our approach to mapping OPM schemas into relational definitions and queries is to use an intermediate EERM level, so that OPM schemas are napped first into EERM schemas and queries, and then EERM schemas and queries are mapped into relational database schema definitions and queries. This approach allows reducing the development of a complex OPM to relational DBMS mapping to a simpler OPM to EERM mapping, while taking advantage of the existing EERM to relational DBMS translation tools $[8,11]$ for generating relational database definitions and queries from EERM schemas and queries.

The complete specification of the OPM schema mapping procedure and examples can be found in [2]. Informally, mapping OPM into EERM consists of mapping every OPM object or protocol class into an entity-set, and of incrementally constructing COQL queries associated with these entity-sets, that express the construction (retrieval) of OPM objects and protocols from EERM entities 
and relationships. Depending on their type (primitive, abstract, simple, composite, etc.), non-derived attributes of object or protocol classes are mapped into EERM attributes, direct entity-set associations, or relationship-sets. Derived attributes are not mapped into EERM schema components (with the exception of some inverse attributes) and entail only modifying COQL queries. For each (object or protocol) class, the mapping generates a COQL query for retrieving the instances in this class, including the values for all their non-derived, derived, and inherited attributes. The mapping also generates a metadatabase that contains information on the cor respondence between the components of the OPM schema and the components of the generated EER schema and COQL queries. The OPM browsing and query tools we plan to develop will be based on this metadatabase.

The OPM schema translator has been developed on Sun SPARCstations in $\mathrm{C}++$ using Lext+ and Yacct+.

\section{Summary and Future Plans}

We have briefly discussed the development of data management tools that allow specifying genornic database structures. These tools are based on the Object-Protocol Model (OPM) developed by us and target commercial relational database management systems. These tools have been applied to the development of a genomic database supporting the sequencing project at University of Washington, Seattle.

We are currently developing OPM data entry and browsing tools. These tools will provide facilities for: (i) inserting, deleting, and updating objects and protocols; (ii) selecting and displaying objects and protocols that satisfy certain conditions; (iii) browsing through selected sets of objects and protocols; (iv) recursively displaying, for a given object or protocol, related objects or protocols

We also plan to develop a more complex OPM query language and a query tool based on this language. This tool will allow querying genomic databases in terms of objects and protocols, and will consist of two main components: (i) an OPM-based graphical interface will allow users to browse through OPM schema specifications and incrementally specify queries in terms of object and protocols; and (ii) a translator will map OPM queries into COQL queries, and subsequently into SQL queries.

The data management tools we develop are currently targeting the Sybase DBMS, mainly becalise Sybase is widely used in molecular biology laboratories and centers worldwide. We recognize that relational databases are cumbersome for implementing genomic databases. Since object-oriented DBMSs are more amenable to represent complex protocols and DNA sequences and provide mechanisms for incorporating application-specific (e.g., sequence alignment) operators, we plan to use such DBMSs for genomic databases. We will experiment with one of the $\mathrm{C}++$ based object-oriented DBMSs, such as Object Store, and will extend our tools in order to ensure an easy transfer of genomic databases 
to these DBMSs.

Acknowledgements. The OPM schema editor was implemented by Ofer BenShachar, Francis Pang, and Carol Jean Smith. SDT was implemented by Weiping Fang and Jun Wang. The COQL translator has been implemented by Ernest Szeto. We greatly appreciate their excellent work.

\section{References}

[1] Chen, I.A., and Markowitz, V.M., The Object-Protocol Model, Lawrence Berkeley Laboratory Technical Report LBL-32738, 1993.

[2] Chen, I.A., and Markowitz, V.M., Mapping Object-Protocol Schemas into Extended Entity-Relationship Schemas and Queries, Lawrence Berkeley Laboratory Technical Report LBL-33048, 1993.

[3] Chen, I.A., Markowitz, V.M., Ben-Shachar, O., and Pang, F., OPM Schema Editor 1.1: A Graphical Editor for Object-Protocol Schemas, Lawrence Berkeley Laboratory Technical Report LBL-33410, 1993.

[4] Hammer, M., and McLeod, D., Database Description with SDM: A Semantic Database Model, ACM Transactions on Database Systems, 6, 3, (1981), pp. 351-386.

[5] Hull, R., and King, R., Semantic Database Modeling: Survey, Applications, and Research Issues, Computing Surveys 19, 3 (1987), pp. 201-260.

[6] loannidis, Y.E., and Livny, M., MOOSE: Modeling Objects in a Simulation Environment, Information Processing 89, G.X. Ritter (ed), Elsevier Science Publishers B.V., 1989, pp. 821-826.

[8] Markowitz, V.M., Fang, W., and Wang, J., SDT 6.0. A Schema Definition and Translation Tool for Extended Entity-Relationship Schemas, Lawrence Berkeley Laboratory technical Report LBL-27843, 1993.

[9] Markowitz, V.M., Lewis, S., McCarthy, J., Olken, F., and Zorn, M., Data Management for Genomic Mapping Applications: A Case Study, Proc. of the 6th International Conference on Scientific and Statistical Database Management, 1992, pp. 45-57.

[10] Markowitz, V.M., and Shoshani, A., Representing Extended EntityRelationship Structures in Relational Databases: A Modular Approach, ACM Transactions on Database Systems, 17, 3, (1992), pp. 423-464.

[11] Markowitz, V.M., and Shoshani, A., Object Queries over Relational Databases: Language, Implementation, and Applications, Proc. of the 9th International Conference on Data Engineering, 1993, pp. 71-80. 

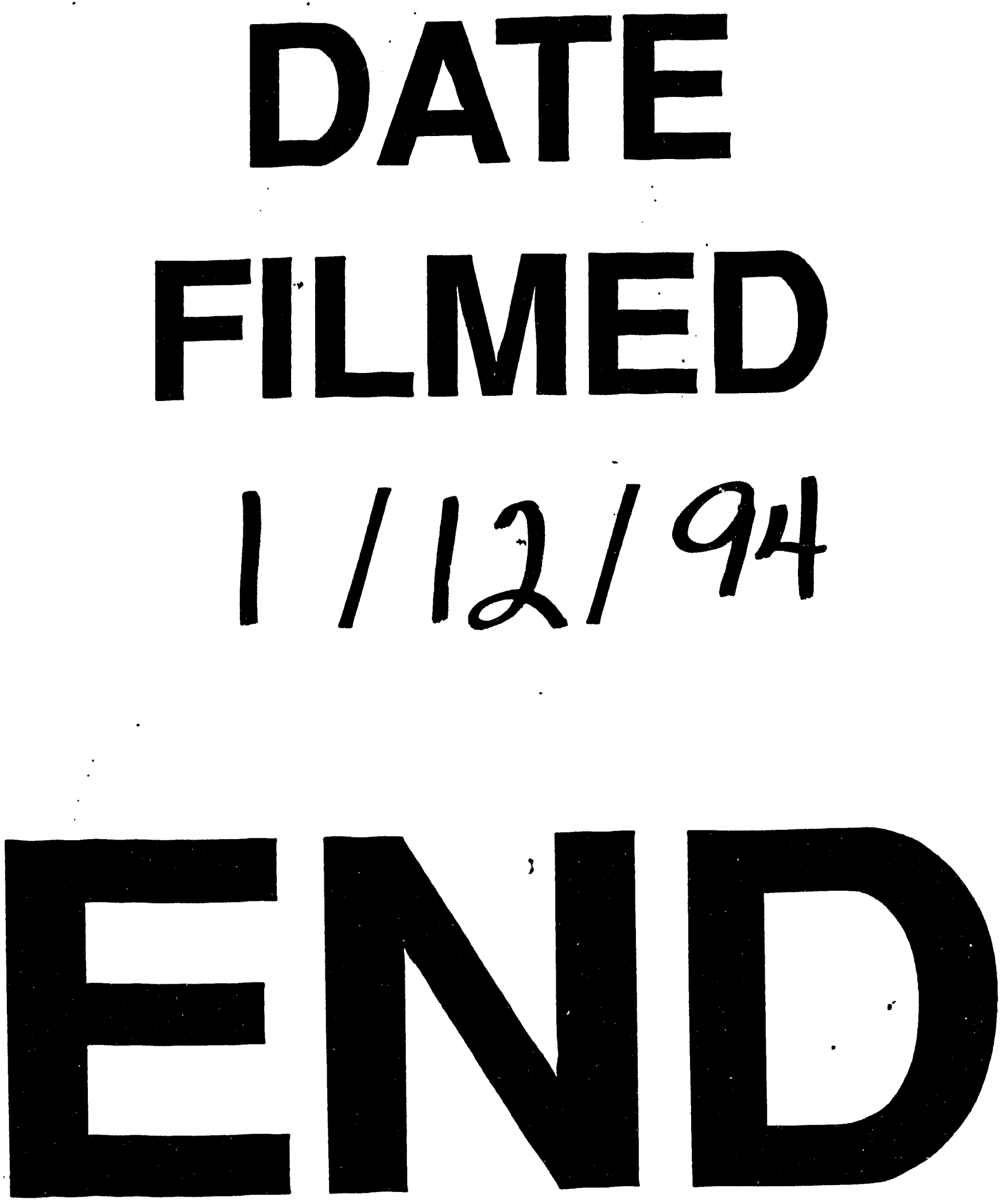
$$
\text { - }
$$

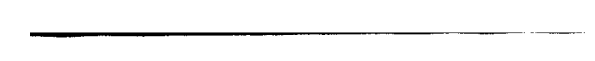

